\title{
High voltage electrical burn resulting in upper limb amputation
}

\begin{abstract}
Electrical injuries are relatively common, almost always accidental and generally preventable. The direct effect of electrical current, the conversion of electrical energy into thermal energy and blunt mechanical trauma can result in tissue destruction and organ dysfunction. Appropriate therapy includes trauma treatment and a detailed physical assessment. Various surgical interventions, including fasciotomy, grafting and even amputation, in addition to long-term supportive care.
\end{abstract}

Keywords: burns, electric; amputation; emergencies

Volume 5 Issue I - 2020

\section{Pedro Nogarotto Cembraneli,', Julia Brasileiro de Faria Cavalcante,' Renata Brasileiro de Faria Cavalcante, ${ }^{2}$ Volmer Fernandes Valente Junior, José Edison da Silva Cavalcante ${ }^{3}$}

'Medical Sciences Course, Health Sciences School, Faculdade Ceres (FACERES), São José do Rio Preto, Brazil

${ }^{2}$ Neurosurgeon, Member of the Brazilian Society of Neurosurgery, Santa Mônica Hospital, Brazil

${ }^{3}$ Neurosurgeon, PhD in Neurosurgery, Member of the Brazilian Society of Neurosurgery, Professor at Santa Mônica Hospital, Brazil

Correspondence: Pedro Nogarotto Cembraneli, Medica Sciences Course, Health Sciences School, Faculdade Ceres (FACERES), São José do Rio Preto, SP, Brazil, Tel (+55) I298। I69654, Email pedrocembranell@hotmail.com

\section{Introduction}

As burns have been a major public health problem, it is not only the number of injuries caused and the number of complications, but also the number of relevant sequences that mark or are burned. ${ }^{1}$ Electrical injuries are almost always accidents and preventable. High voltage injuries are caused by exposure $>1000$ volts and are relatively rare when compared to other causes of burns caused by emergency units. ${ }^{2}$ The direct effect of electrical current, a conversion to thermal energy and mechanical trauma that can cause tissue destruction and organ dysfunction. These accidents often have long periods of hospitalization, with various surgical approaches. Mortality in the acute period is often a result of cardiac and respiratory arrest, shock, renal failure and sepsis. ${ }^{3}$ Thus, early intervention, still without a hospital environment, is essential to obtain the expected results of functionality, healing and restoration of patients' physical and emotional health.

\section{Case report}

Male, 32, bricklayer, admitted to the emergency room brought by the fire department with a history of trauma after a high voltage electrical injury related to work while working on top of a construction site. Patient lost his balance and accidentally grabbed a 14,000 V cable with his left hand. He fell from approximately 3 meters in height and suffered blunt head trauma resulting in loss of consciousness. Upon arriving at the hospital, the patient was alert, oriented and hemodynamically stable. The patient had burns visible on $3 \%$ of the total body surface area involving the distal forearm and the left hand. (Figure 1A), with third degree burns. Computed tomography of the head did not show any lesions. In addition, he presented myoglobinuria and a creatine kinase of $34 \times 10^{3} \mathrm{U} / \mathrm{L}$, and abundant intravenous hydration was started. Within hours, the patient evolved with elevated compartment pressures and was immediately taken to the operating room for escarotomies and left upper limb fasciotomies (Figure 1B). The patient evolved with tissue necrosis that extended from the forearm to the level of the wrist, involving both the extensor and flexor compartments (Figure 1C). Despite escarotomies, fasciotomies and serial debridement, the patient's left upper limb became progressively congested with poor doppler signals and low $\mathrm{O} 2$ saturation within each digit. It was necessary to perform the amputation from the distal third of the left arm (Figure 1D). The patient was kept in the hospital for 21 days to correct hydroelectrolytic disorders and was referred to the rehabilitation center.

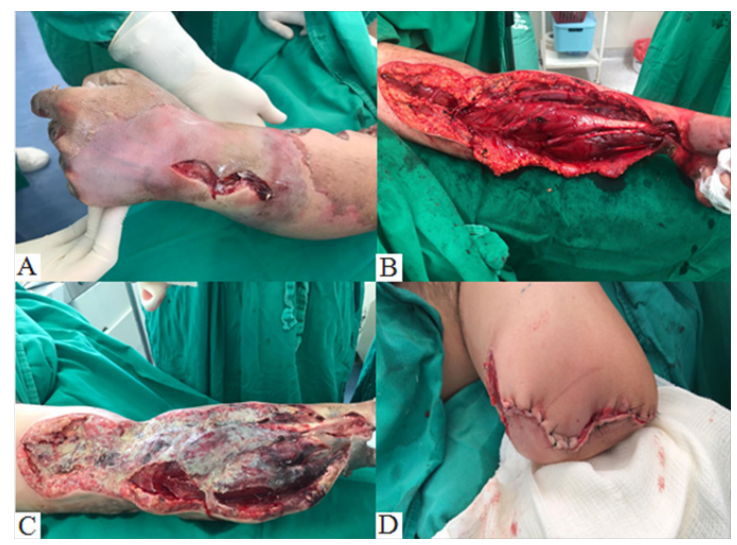

Figure I A- burns visible on $3 \%$ of the total body surface area involving the distal forearm and the left hand. B- escarotomies and left upper limb fasciotomies. C- tissue necrosis of the forearm to the level of the wrist. Damputation from the distal third of the left arm. 


\section{Discussion}

The burn injury is not only a medical emergency, but it triggers physical, psychological and financial problems for the patient, his family and society. The effects of major burns can be considered irreparable in all areas of the patient's life. ${ }^{1}$

High voltage injuries are characterized by a low incidence of electrical injuries in general and can cause high morbidity, when compared to low voltage injuries. ${ }^{4}$

In the initial evaluation, many associated injuries may exist, orthopedic injuries, explosion injuries; cognitive problems, such as sleep disorders, lack of memory, attention deficit, headache, irritability; paresthesias, depression and muscle spasms, inhalation injuries, cataracts, gastrointestinal injuries; vascular insufficiency, mainly compartment syndrome, disseminated intravascular dissemination; neurological injuries, reflex sympathetic dystrophy, cardiac and renal injuries. ${ }^{5,6}$

Early serial debridement of extensive necrosis or amputation of limbs is performed in order to decrease the likelihood of infection and decrease the amount of toxins and myoglobin released by the injured tissue that could be absorbed into the bloodstream. Indications for amputation are signs of deep tissue injury or septic focus. ${ }^{7}$

The greatest sequelae include motor limitation (difficulty walking and using prostheses), permanent neurological injuries (paresthesias, paresis and dizziness) and the patient's aesthetic appearance. It is worth mentioning that protection through adequate protective equipment and exhaustive educational campaigns remains the best form of prevention and is the most important determining factor in reducing the number of sequelae from electrical burns. ${ }^{8}$

\section{Conclusion}

The patient must be approached according to the protocols of advanced cardiac life support and advanced trauma support. Vigorous volume replacement, identification and treatment of compartment syndrome, appropriate burn care and tetanus prophylaxis are the most important measures.

\section{Funding}

None.

\section{Acknowledgments}

None.

\section{Conflicts of interest}

The authors declare no conflicts of interest.

\section{References}

1. Albuquerque MLL, Silva GPF, Diniz DMSM, et al. Analysis of burn patients with motor sequelae in a qualified hospital in Fortaleza-CE. Rev Bras Queimaduras. 2010;9(3):89-94.

2. Tintinalli, Judith E. Emergency medicine: a comprehensive study guide (emergency medicine (Tintinalli)). New York: McGraw-Hill Companies. 1374-1386.

3. Silva JAC, Vendramin FS, Martins MM, et al. Epidemiology, main complications and mortality of patients treated at a burn treatment center in the Amazon. Rev Bras Cir Plást. 2018;33(1):104-109.

4. Leonardi DF, Laporte GA, Tostes FM. Limb amputation due to high voltage electrical burn. Rev Bras Queimaduras. 2011;10(1):27-29.

5. Celebi A, Gulel O, Cicekcioglu H, et al. Myocardial infarction after an electric shock: a rare complication. Cardiol J. 2009;16(4):362.

6. Cherington M. Neurologic manifestations of lightning strikes. Neurology. 2003;60(2):182.

7. Davis C, Engeln A, Johnson E, et al. Wilderness medical society practice guidelines for the prevention and treatment of lightning injuries. Wilderness Environ Med. 2012;23(3):260.

8. Oliveira RA, Bersan ML, Dupin AE, et al. Approach to electrical burn in upper limb. Relato de Caso Rev Bras Queimaduras. 2013;12(3):187191 